\title{
Wie bewerten chronisch Kranke die organisatorischen und interpersonellen Aspekte ihrer haus- und fachärztlichen Versorgung im Vergleich? Ergebnisse einer deutschlandweiten Befragung
}

\author{
How do Chronically III Patients Rate Medical Care by their GPs and Specialists? \\ Results of a Germany-wide Survey
}

Autoren

Institut
J. Röttger, R. Busse

Fachgebiet Management im Gesundheitswesen, Gesundheitsökonomisches Zentrum Berlin, Technische Universität Berlin

\section{Schlüsselwörter \\ - Health System Responsiveness \\ - Chronisch Kranke \\ - Hausarzt \\ - Facharzt \\ - ambulante Versorgung \\ - Patientenorientierung}

Key words

- health system responsiveness

- chronically ill

- general practitioner

- specialist care

- ambulatory care

- patient orientation

Bibliografie

Dol http://dx.doi.org/

10.1055/s-0041-110669

Online-Publikation: 16.2.2016

Gesundheitswesen 2016;

78 (Suppl. 1): e89-e96

(c) Georg Thieme Verlag KG

Stuttgart · New York

ISSN 0949-7013

Korrespondenzadresse

\section{Julia Röttger, MSc}

Fachgebiet Management im

Gesundheitswesen

Gesundheitsökonomisches

Zentrum Berlin

Technische Universität Berlin

julia.roettger@tu-berlin.de

\section{Zusammenfassung}

$\nabla$

Einleitung: Die Fähigkeit eines Gesundheitssystems auf die legitimen Erwartungen der Bevölkerung hinsichtlich der organisatorischen und interpersonellen Aspekte der Versorgung einzugehen, die sogenannte Health System Responsiveness, wird als eine Kernfähigkeit von Gesundheitssystemen angesehen. Während in vielen Studien die Responsiveness im ambulanten Sektor erhoben wurde, gibt es bisher kaum Informationen zu Unterschieden in der Bewertung der haus- und fachärztlichen Versorgung. Methodik: Mittels einer schriftlichen Befragung wurden 51998 chronisch kranke Versicherte (Koronare Herzkrankheit und/oder Diabetes Typ II) der Techniker Krankenkasse hinsichtlich ihrer Erfahrungen mit haus- und fachärztlicher Versorgung befragt. Die Erfahrungen mit der Versorgung, getrennt nach Haus- und Fachärzten, wurden mit einer adaptierten Version des Health System Responsiveness Fragebogens der WHO erfasst. Die Unterschiede in der Bewertung wurden mittels McNemar-Test analysiert.

Ergebnisse: Die Antworten von 13685 Versicherten konnten in die Analysen eingeschlossen werden. Die Befragten bewerteten ihre Versorgung überwiegend positiv. Über alle abgefragten Dimensionen hinweg wurden die Hausärzte statistisch signifikant besser bewertet als die Fachärzte. Die stärksten Unterschiede zeigten sich dabei bei den Bewertungen der Wartezeit, der Einbeziehung in die Entscheidungsfindung sowie der Koordination der Behandlung.

Schlussfolgerung: Insgesamt sind die befragten Versicherten überwiegend zufrieden mit ihrer haus- und fachärztlichen Versorgung. Insbesondere bei der fachärztlichen Versorgung zeigen sich jedoch auch Bereiche mit Optimierungsbedarf.

\section{Abstract \\ $\nabla$}

Introduction: The ability of health systems to respond to the legitimate expectations of the population regarding interpersonal and organizational aspects of healthcare - the so-called Health System Responsiveness - is considered a key competence of health systems. While various studies have assessed the responsiveness of ambulatory care, information on differences between care provided by general practitioners (GP) and specialists is still scarce.

Methods: By means of a postal survey, 51998 chronically ill persons (type 2 diabetes and/or coronary heart disease) insured by a statutory health insurance body (Techniker Krankenkasse) were surveyed regarding their experiences with GP and specialist care. An adapted version of the WHO Health System Responsiveness questionnaires was used for assessing data. Differences in the rating of specialist and GP care were analyzed using McNemar's test.

Results: Responses from 13685 patients were included in data analyses. Overall, ambulatory care was rated mostly as positive. Across all dimensions of health system responsiveness, GP care was rated statistically significantly as more positive than specialist care. Considerable differences were found in the evaluation of waiting times, involvement in decision-making and coordination of care.

Conclusion: Overall, the surveyed persons were mostly satisfied with their GP and specialist care. However, some aspects of specialist care need to be optimized. 


\section{Einleitung \\ 7}

Neben dem Erhalt und der Förderung von Gesundheit sowie der finanziellen Absicherung im Krankheitsfall, gilt die Patientenbzw. Nutzerorientierung in der nationalen und internationalen Diskussion als ein hochgradig relevantes Attribut von Gesundheitssystemen [1,2]. Entsprechend wird diese als ein Aspekt in der Bewertung der Leistungsfähigkeit von Gesundheitssystemen genutzt $[1,3,4]$. Eine Möglichkeit Patientenorientierung zu erfassen, bietet das Konzept der Health System Responsiveness der Weltgesundheitsorganisation (WHO). Unter der Responsiveness eines Gesundheitssystems wird die Fähigkeit des Gesundheitssystems, auf die legitimen Erwartungen der Bevölkerung hinsichtlich interpersoneller und organisatorischer Aspekte der Versorgung einzugehen, verstanden. Hierzu zählt zum einen die Bewertung von Erfahrungen im direkten Kontakt mit dem Gesundheitssystem (z.B. verständliche Kommunikation, respektvoller Umgang usw.) und zum anderen Erfahrungen hinsichtlich des Zugangs zu gesundheitlicher Versorgung [5].

Die Responsiveness eines Gesundheitssystems wurde international in vielen Studien erfasst (u.a. [6-11]). Dabei wurde zwar zumeist zwischen ambulanter und stationärer Versorgung unterschieden, weitergehende Unterschiede in der ambulanten Versorgung, z.B. hinsichtlich fach- und hausärztlicher Versorgung, wurden jedoch bisher wenig betrachtet. Oftmals wird die ambulante Versorgung ausschließlich zusammengefasst dargestellt, so z. B. in den Studien der WHO oder den Health Care Quality Indikatoren der OECD [12-14].

Die vorhandenen Studien, die zwischen haus- und fachärztlicher Versorgung unterscheiden, fokussieren dabei häufig spezifische Patientengruppen (z. B. onkologische Patienten [15]) oder erfassen nur wenige Aspekte der Versorgung. Diese werden dann zudem häufig entweder für die haus- oder für die fachärztliche Versorgung abgefragt [16].

Somit liegen bisher nur wenige vergleichende Informationen $\mathrm{zu}$ der Responsiveness der haus- und fachärztlichen Versorgung aus Sicht von Patienten vor. An diesem Punkt setzt die vorliegende Studie an. Anhand einer deutschlandweiten Befragung wird untersucht, inwieweit sich die aus Sicht von chronisch kranken Personen wahrgenommene Responsiveness der haus- und fachärztlichen Versorgung unterscheidet.

\section{Methodik}

$\nabla$

Die Daten für die Analysen stammen aus dem Projekt Exploring health system responsiveness in ambulatory care and disease management and its relation to other aspects of health system performance (RAC). Im Oktober 2013 wurden im Zuge von 2 Sub-Studien des Projekts 51998 Versicherte der Techniker Krankenkasse hinsichtlich der von ihnen wahrgenommenen Responsiveness der haus- und fachärztlichen Versorgung befragt. Da das Hauptziel des Projekts die Analyse der Determinanten (u.a. Teilnahme an einem DMP, Inanspruchnahme von Gesundheitsleistungen) der berichteten Health System Responsiveness ist, wurde angestrebt einen Datensatz zu erhalten, der sowohl Befragungs- als auch Routinedaten der Krankenkasse auf Individualebene enthält. In einer Sub-Studie des Projekts lag der Fokus auf Personen mit Koronarer Herzkrankheit (KHK), während in der zweiten Sub-Studie Personen mit einem Diabetes mellitus Typ II (DM2) befragt wurden. Auf Grundlage von Routinedaten wurden für jede Sub-Studie 25999 Versicherte ausgewählt, (i) die an KHK und/oder DM2 erkrankt sind und (ii) entweder bereits in das entsprechende Disease Management Programm (DMP) eingeschrieben sind oder die Voraussetzungen für eine Einschreibung in das entsprechende DMP erfüllen, aber nicht eingeschrieben sind.

Den ausgewählten Versicherten wurden ein Fragebogen sowie eine Einverständniserklärung für die Verknüpfung der Befragungsdaten mit ausgewählten Routinedaten zugesandt. Wenn beide Dokumente ausgefüllt zurückgeschickt wurden, wurden die Daten anschließend verknüpft und in die späteren Auswertungen einbezogen.

Der eingesetzte Fragebogen erfasste unter anderem die wahrgenommene Health System Responsiveness der haus- und fachärztlichen Versorgung. Die Fragen deckten dabei 7 von der WHO entwickelte Dimensionen der Health System Responsiveness ab [5] sowie 2 Dimensionen, um die das WHO Konzept auf Grundlage von Fokusgruppen erweitert wurde (Koordination und Vertrauen) [17]. Die insgesamt 17 Fragen zur Responsiveness bezogen sich dabei jeweils auf den letzten Haus- und Facharztbesuch innerhalb der letzten 12 Monate. Bei der Bewertung des Facharztbesuches wurde nicht nach verschiedenen Facharztrichtungen unterschieden. Alle Fragen wurden 2-mal gestellt, einmal zum Haus- und einmal zum Facharztbesuch und verfügten jeweils über 5 Antwortkategorien von „sehr gut“ bis „sehr schlecht“ ( $\bullet$ Tab. 1). Dem Vorgehen der WHO entsprechend, wurden für die späteren Auswertungen die 5 Antwortkategorien in je 2 Kategorien zusammengefasst (sehr gut und gut/mittelmäßig, schlecht und sehr schlecht) [18].

Zusätzlich zu der Bewertung der letzten Arztbesuche, wurde über 3 Fragen ein Verzicht auf gesundheitliche Versorgung erfasst ( $\bullet$ Tab. 1).

Die Studie erhielt ein positives Votum durch die zuständige Ethikkommission. Für eine detaillierte Beschreibung der Stichprobenselektion, Erhebungsinstrumente und Datenerfassung siehe [19].

In die Analysen hinsichtlich der Unterschiede zwischen der Bewertung der fach- und hausärztlichen Versorgung wurden nur Fälle einbezogen, die sowohl einen Haus- als auch Facharztbesuch innerhalb der letzten 12 Monate berichteten. Bei dem Vergleich der einzelnen Fragen wurden wiederum nur Fälle einbezogen, die jeweils die beiden zueinander gehörenden Fragen (z.B. Wartezeit Facharzttermin und Wartezeit Hausarzttermin) beantwortet haben. Dies führt zwar zu einem höheren Ausschluss an Fällen; Unterschiede in den Bewertungen können somit aber nicht durch Unterschiede der Befragten bedingt sein. Die statistische Signifikanz der Unterschiede wurde mittels McNemar-Test analysiert. Dabei wurde über die BonferroniKorrektur für multiples Testen adjustiert; das Signifikanzniveau von $\alpha=0,05$ wurde entsprechend auf $\alpha=0,001$ gesetzt.

\section{Ergebnisse}

$\nabla$

\section{Deskriptive Beschreibung der Stichprobe}

Der Netto-Rücklauf, nach Abgleich der eingegangen Fragebögen mit den zurückgeschickten Einverständniserklärung für die Datenverknüpfung sowie dem Ausschluss von unvollständigen Fragebögen, betrug 15565 Fälle (29,9\%). Von den 15565 Fällen hatten 13685 (87,9\%) sowohl einen Haus- als auch einen Facharztbesuch innerhalb der letzten 12 Monate berichtet. Diese Fälle bilden die finale Stichprobe, welche für alle weiteren Analysen genutzt wurde ( $\bullet$ Abb. 1). 
Tab. 1 Fragen zur Erfassung der Responsiveness der haus- und fachärztlichen Versorgung sowie des Verzichts auf Versorgung.

\section{Frage/Dimension}

Wenn Sie an die letzten 12 Monate denken - Haben Sie in dieser Zeit irgendeine Art von medizinischer Versorgung bei einem Hausarzt (Allgemeinmediziner/ Internist) erhalten? (Ja/Nein)

Bitte denken Sie erneut an die letzten 12 Monate - Haben Sie in dieser Zeit irgendeine Art von medizinischer Versorgung bei einem niedergelassenen Facharzt (z. B. Orthopäde, Augenarzt, Hautarzt,...) erhalten? (Ja/Nein)

Wie würden Sie bei Ihrem letzten Hausarztbesuch.../Wie würden Sie bei Ihrem letzten Facharztbesuch... (Antwortkategorien aller Folgefragen: sehr gut/gut/ mittelmäßig/schlecht/sehr schlecht)

Sofortige Aufmerksamkeit

...die Wartezeit auf einen Termin (d.h. die Zeit zwischen der Anfrage nach einem Termin bis zum tatsächlichen Termin) bei Ihrem Arzt/ Ihrer Ärztin bewerten?

...die Wartezeit im Wartezimmer bewerten, bis Sie an der Reihe waren?

Wahlfreiheit

...Ihre Möglichkeiten bewerten, sich die Sie behandelnde Arztpraxis/ Person selbst aussuchen zu können? ${ }^{1}$

Respektvoller Umgang

...Ihre Erfahrungen bewerten, inwiefern Sie respektvoll empfangen und mit Ihnen respektvoll gesprochen wurde? ${ }^{1}$

....thre Erfahrungen bewerten, inwiefern Sie in der ärztlichen Praxis freundlich behandelt wurden?

...Ihre Erfahrungen bewerten, inwiefern Sie während der ärztlichen Behandlung die volle Aufmerksamkeit erhalten haben?

...Ihre Erfahrungen bewerten, inwiefern Sie in der ärztlichen Praxis mit Einfühlungsvermögen behandelt wurden?

Kommunikation

....Ihre Erfahrungen bewerten, inwiefern Ihnen von den Ärzten/ dem Personal Dinge verständlich erklärt wurden? ${ }^{1}$

Autonomie/Einbeziehung in Entscheidungen

....hre Erfahrungen bewerten, inwiefern Sie in Entscheidungen, die Ihre Behandlung betrafen, miteinbezogen wurden? ${ }^{1}$

Vertraulichkeit

....Ihre Erfahrungen bewerten, inwieweit sichergestellt wurde, dass Sie mit der Sie behandelnden Person vertraulich sprechen konnten? ${ }^{1}$

....Ihre Erfahrungen bewerten, inwieweit sichergestellt wurde, dass Sie am Empfang der Arztpraxis vertraulich sprechen konnten?

Qualität der Ausstattung

...die Sauberkeit der Räume (einschließlich der Toiletten) bewerten? ${ }^{1}$

...die Ausstattung der Räume (z. B. ausreichend Stühle im Wartezimmer usw.) bewerten?

Koordination

...Ihre Erfahrungen bewerten, inwiefern Ihre behandelnden Ärzte sich untereinander abgesprochen haben bzw. Informationen zwischen Ärzten weitergegeben wurden?

....hre Erfahrungen bewerten, inwiefern Ihre behandelnden Ärzte Ihren Behandlungsablauf kannten (z. B. wussten, wann eine Kontrolluntersuchung notwendig war)?

Vertrauen

....hre Erfahrungen bewerten, inwiefern Ihre ärztliche Behandlung ausschließlich Ihr gesundheitliches Wohlergehen zum Ziel hatte (d. h. nicht von anderen Interessen geleitet war)?

...Ihre Erfahrungen bewerten, inwiefern Ihre ärztliche Beratung ausschließlich Ihr gesundheitliches Wohlergehen zum Ziel hatte (d. h. nicht von anderen Interessen geleitet war)?

Verzicht auf Versorgung

Haben Sie in den letzten 12 Monaten jemals auf eine medizinische Versorgung verzichtet, obwohl diese aus Ihrer Sicht notwendig war? (Ja/Nein)

Auf welche Arten der medizinischen Versorgung haben Sie verzichtet?

Medikamente (Ja/Nein)

Termin bei einem Facharzt (Ja/Nein)

Termin bei einem Hausarzt (Ja/Nein)

Vorsorgeuntersuchung (Ja/Nein)

Andere diagnostische Untersuchungen (Ja/Nein)

Operativer Eingriff (Ja/Nein)

Sonstige: (Ja/Nein und Freitext)

Und aus welchen Gründen haben Sie auf die Versorgung verzichtet?

Keine ausreichenden finanziellen Mittel (Ja/Nein)

Die Wartezeit auf einen Termin war zu lang (Ja/Nein)

Der Weg zur Arztpraxis war zu lang/ zu beschwerlich (Ja/Nein)

Kein geeigneter Facharzt stand zur Verfügung (Ja/Nein)

Sonstige: (Ja/Nein und Freitext)

${ }^{1}$ original WHO Fragestellung World Health Survey 2002-2003 (Quelle [5]).

- Tab. 2 gibt einen Überblick über die soziodemografischen Charakteristika der Befragten in der finalen $(n=13685)$ sowie in der gesamten Stichprobe $(n=15565)$. Die gesamte Stichprobe setzt sich zu 54,4\% aus Teilnehmern zusammen, die für die Sub-Studie KHK angeschrieben wurden und zu $45,6 \%$ aus Teilnehmern der Sub-Studie DM2. In der finalen Stichprobe ist die Zusammensetzung 54,9\% KHK und 45,1\% DM2.

Die Befragten in der finalen Stichprobe waren im Durchschnitt 69,7 (SD: 10,0) Jahre alt. 71,7\% der Befragten waren männlich und die Mehrheit der Befragten befand sich zum Zeitpunkt der Befragung in Altersrente ( $\bullet$ Tab. 2). Die Mehrheit der Befragten, $61,5 \%$, war in ein DMP eingeschrieben ( $\odot$ Tab. 3). Nur ca. 10,5\% der Teilnehmer hatten laut stationärer und ambulanter Diagnosedaten der Quartale II/2012 bis III/2013 keine der Ko-Morbiditäten der ambulanten Erweiterung des Charlson Index ${ }^{1}$ [20]. Der

\footnotetext{
${ }^{1}$ Diabetes, Herzinsuffizienz und Myokardinfarkt wurden bei der Berechnung des Charlson Index nicht mitgezählt, wenn die Befragten für die entsprechende Sub-Studie angeschrieben wurden
} 


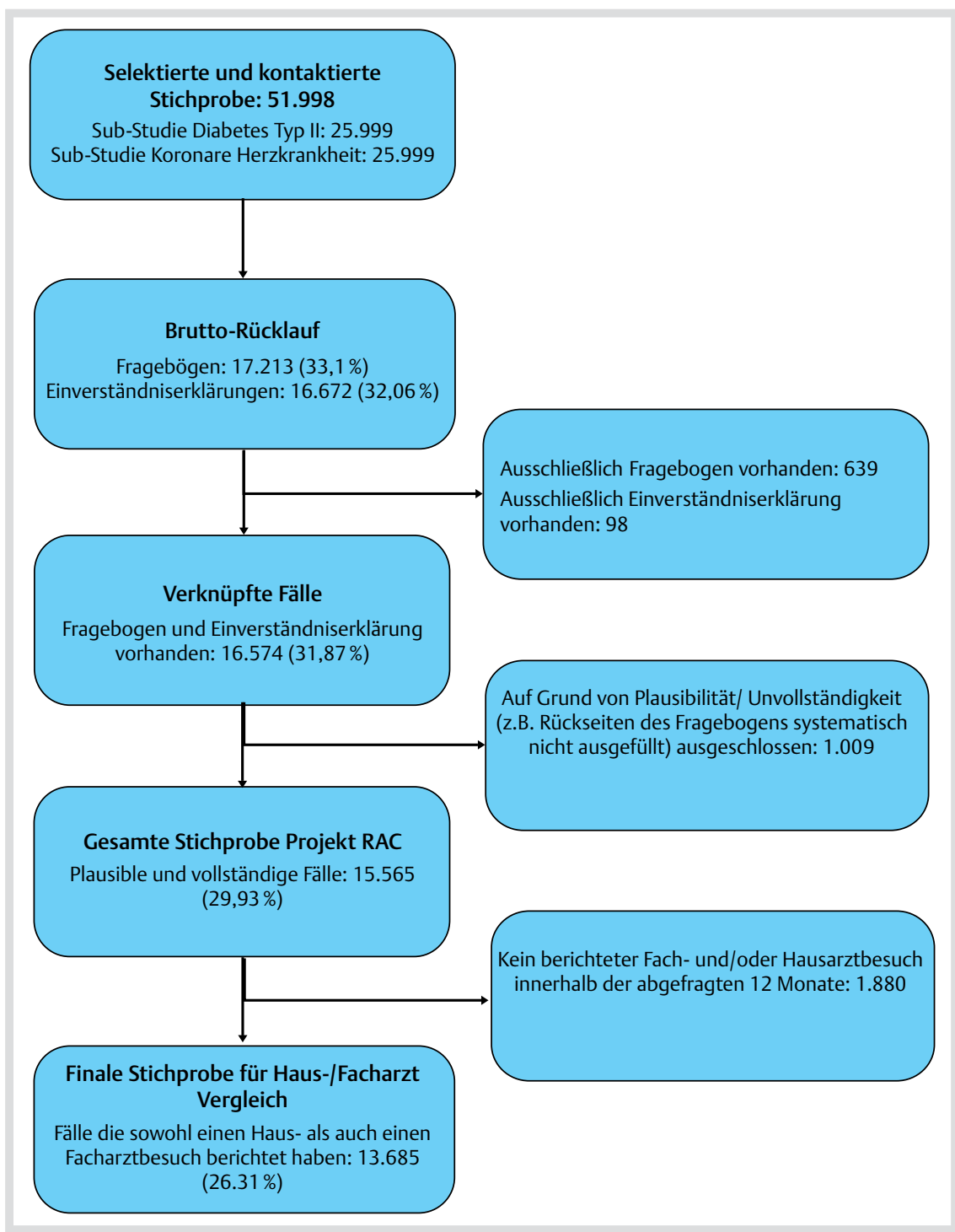

Abb. 1 Rücklauf und Fallausschluss.

Großteil der Studienteilnehmer wurde in den betrachteten sechs Quartalen nicht stationär behandelt ( $\bullet$ Tab. 3).

\section{Bewertung der Responsiveness der hausärztlichen und fachärztlichen Versorgung}

Die Anzahl der Personen, die die zusammengehörenden Fragen sowohl für den letzten Besuch bei dem Haus- als auch bei dem Facharzt beantwortet haben, liegt zwischen 12761 (Koordination zwischen Ärzten) und 13289 (volle Aufmerksamkeit während der Behandlung) ( $\bullet$ Abb. 2). Bezogen auf die Anzahl der eingeschlossenen Fälle, entspricht dies einer Responserate zwischen 93,2 und $97,1 \%$.

Bei der hausärztlichen Versorgung wurden die Aspekte „Sauberkeit der Räume“ und „Freundlichkeit“ mit 96,2 bzw. 95,5\% positiven Antworten (Antwortkategorie „gut“ oder „sehr gut") am besten bewertet. Am schlechtesten bewertet wurden hingegen die Bereiche „Vertraulich sprechen am Empfang“ sowie „Wartezeit im Wartezimmer“ mit 59,5 bzw. 70,4\% positiven Bewertungen.

Bei der fachärztlichen Versorgung wurden ebenfalls die Bereiche „Sauberkeit der Räume“ und „Freundlichkeit“ am besten bewertet, hier antworteten 94,9 bzw. 90,0\% mit "sehr gut“ oder "gut“. Am schlechtesten wurden hingegen die Bereiche „Wartezeit
Termin“ und „Wartezeit Wartezimmer“ bewertet, mit 52,4 bzw. $54,2 \%$ positiven Antworten.

Über alle Kategorien hinweg wurden die Hausärzte statistisch signifikant besser bewertet als die Fachärzte. Die stärksten Unterschiede zeigten sich dabei bei den Bewertungen der Wartezeit, der Einbeziehung in die Entscheidungsfindung sowie der Koordination, hierbei insbesondere bei der Kenntnis über den Behandlungsablauf. Die kleinsten Unterschiede zeigten sich hingegen bei der Sauberkeit und Ausstattung der Räume sowie bei der Möglichkeit vertraulich am Empfang sprechen zu können. Bei letzterem wurden sowohl die Haus- als auch die Fachärzte im Vergleich $\mathrm{zu}$ allen anderen Bereichen relativ schlecht bewertet.

Im Vergleich zwischen den Sub-Studien ( $\bullet$ Tab. 4 im Internet) zeigten sich geringe Unterschiede in der Bewertung der Versorgung zwischen Befragten der Gruppe KHK und DM2, wobei die Gruppe KHK ihre Versorgung tendenziell etwas negativer bewertete als die Gruppe DM2.

\section{Berichteter Verzicht auf haus- und/oder fachärztliche Versorgung}

Von den 13685 Befragten, die sowohl einen Haus- als auch Facharztbesuch innerhalb der letzten 12 Monate berichteten, gaben 1920 Personen $(14,0 \%)$ an, innerhalb der letzten 12 
Tab. 2 Soziodemographische Beschreibung der finalen und gesamten Stichprobe (alle Angaben in \%).

\begin{tabular}{|c|c|c|}
\hline & $\begin{array}{l}\text { Finale } \\
\text { Stichprobe } \\
(n=13685)\end{array}$ & $\begin{array}{l}\text { Gesamte } \\
\text { Stichprobe } \\
(n=15565)\end{array}$ \\
\hline Geschlecht (\% männlich) ${ }^{1}$ & 71.7 & 71.4 \\
\hline \multicolumn{3}{|l|}{ Alter $^{1}$} \\
\hline$<=60$ Jahre & 17.0 & 17.8 \\
\hline 61-65 Jahre & 12.4 & 12.5 \\
\hline 66-70 Jahre & 15.6 & 15.4 \\
\hline 71-75 Jahre & 25.3 & 24.8 \\
\hline 76-80 Jahre & 19.3 & 18.9 \\
\hline$>=81$ Jahre & 10.5 & 10.5 \\
\hline \multicolumn{3}{|l|}{ Nettoäquivalenzeinkommen ${ }^{2 \cdot 3}$} \\
\hline Unterste Einkommensgruppe $<=979 €$ & 13.6 & 14.4 \\
\hline $\begin{array}{l}\text { Untere-Mittlere Einkommensgruppe } \\
980 \text { bis } 1633 €\end{array}$ & 43.9 & 43.3 \\
\hline $\begin{array}{l}\text { Obere-Mittlere Einkommensgrup- } \\
\text { pe }>1633 \text { bis }<2449 €\end{array}$ & 23.8 & 23.4 \\
\hline $\begin{array}{l}\text { Höchste Einkommensgruppe = } \\
>2449 €\end{array}$ & 11.2 & 11.1 \\
\hline Keine Angabe & 7.5 & 7.8 \\
\hline \multicolumn{3}{|l|}{ Erwerbstätigkeit ${ }^{2}$} \\
\hline Erwerbstätig (Vollzeit/Teilzeit) & 17.9 & 18.7 \\
\hline $\begin{array}{l}\text { Nicht erwerbstätig (nicht verrentet/ } \\
\text { nicht erkrankt) }\end{array}$ & 2.8 & 2.8 \\
\hline $\begin{array}{l}\text { Nicht erwerbstätig (auf Grund von } \\
\text { Erkrankung) }\end{array}$ & 4.4 & 4.3 \\
\hline Altersrente & 71.9 & 70.9 \\
\hline Keine Angabe & 3.0 & 3.3 \\
\hline \multicolumn{3}{|l|}{ Wohnort (Kreistyp) ${ }^{1}$} \\
\hline Kreisfreie Großstädte & 34.4 & 34.1 \\
\hline Städtische Kreise & 41.6 & 41.8 \\
\hline $\begin{array}{l}\text { Ländliche Kreise mit Verdichtungs- } \\
\text { ansätzen }\end{array}$ & 13.4 & 13.4 \\
\hline Dünn besiedelte ländliche Kreise & 10.6 & 10.6 \\
\hline
\end{tabular}

Monate auf medizinische Versorgung verzichtet zu haben, obwohl der/die Befragte die Versorgung für notwendig hielt. Am häufigsten wurde dabei ein Verzicht auf einen Facharztbesuch berichtet $(\mathrm{n}=1124 ; 8,2 \%)$, gefolgt von einem Verzicht auf Vorsorgeuntersuchungen $(n=659 ; 4,8 \%)^{2}$. Bezogen auf einen Hausarzttermin, gaben 226 Personen $(1,7 \%)$ an, auf diesen verzichtet zu haben ( $\bullet$ Abb. 3). Dabei berichteten 142 Personen sowohl einen Verzicht auf einen Fach- als auch auf einen Hausarzttermin.

Die 1124 Befragten, die einen Verzicht beim Facharzt berichteten, gaben als Gründe für einen Verzicht am häufigsten die Wartezeit auf einen Termin (66,6\%), gefolgt von „es stand kein geeigneter Facharzt zur Verfügung“" (30,2\%) an. Es muss jedoch bedacht werden, dass bei Befragten, die verschiedene Arten des Verzichts (Facharzttermin, operativer Eingriff, usw.) angegeben haben, die Antworten zu den Gründen des Verzichts nicht direkt der Art des Verzichts zugeordnet werden können.

\footnotetext{
${ }^{2}$ In der Gesamtstichprobe $(\mathrm{n}=15565)$ besteht ein ähnliches Bild: 1296 Personen (8,3\%) bzw. 268 Personen (1,7\%) berichteten einen Verzicht auf einen Fach- bzw. Hausarzttermin.
}

Tab. 3 Morbidität und Inanspruchnahme von Gesundheitsleistungen (alle Angaben in \%).

\begin{tabular}{|c|c|c|}
\hline & $\begin{array}{l}\text { Finale } \\
\text { Stichprobe } \\
(n=13685)\end{array}$ & $\begin{array}{l}\text { Gesamte } \\
\text { Stichprobe } \\
(\mathrm{n}=15565)\end{array}$ \\
\hline $\begin{array}{l}\text { Eingeschrieben in DMP (KHK und/oder } \\
\text { DM2) }\end{array}$ & 61.5 & 60.2 \\
\hline \multicolumn{3}{|l|}{ Pflegestufe ${ }^{1}$} \\
\hline Pflegestufe 1 & 2.6 & 2.6 \\
\hline Pflegestufe 2 & 0.8 & 0.9 \\
\hline \multicolumn{3}{|l|}{ Charlson Index } \\
\hline Charlson Index $=0$ & 10.5 & 11.4 \\
\hline Charlson Index $=1$ & 21.3 & 22.0 \\
\hline Charlson Index $=2$ & 19.3 & 19.3 \\
\hline Charlson Index $=3$ & 16.2 & 15.8 \\
\hline Charlson Index $=>4$ & 32.6 & 31.5 \\
\hline \multicolumn{3}{|l|}{$\begin{array}{l}\text { Anzahl der Krankenhausaufnahmen in } \\
\text { sechs Quartalen (II/2012-III/2013) }\end{array}$} \\
\hline Keine Aufnahme & 55.5 & 56.8 \\
\hline 1 Aufnahme & 22.2 & 21.8 \\
\hline 2 Aufnahmen & 11.0 & 10.5 \\
\hline 3 und mehr Aufnahmen & 11.4 & 10.9 \\
\hline \multicolumn{3}{|l|}{$\begin{array}{l}\text { Anzahl der durchschnittlichen Haus- } \\
\text { arztkontakte pro Quartal (II/2012 - } \\
\text { III/2013) }\end{array}$} \\
\hline$<=1$ Kontakt & 3.2 & 3.9 \\
\hline$>1-2$ Kontakte & 13.5 & 14.5 \\
\hline$>2-3$ Kontakte & 27.2 & 27.2 \\
\hline >3-4 Kontakte & 22.5 & 22.1 \\
\hline$>4-6$ Kontakte & 21.4 & 20.7 \\
\hline$>6-8$ Kontakte & 7.4 & 7.0 \\
\hline$>8$ Kontakte & 4.7 & 4.5 \\
\hline \multicolumn{3}{|l|}{$\begin{array}{l}\text { Anzahl der durchschnittlichen } \\
\text { Facharztkontakte pro Quartal (II/2012 } \\
\text { - III/2013)3 }\end{array}$} \\
\hline$<=1$ Kontakt & 16.2 & 20.0 \\
\hline$>1-2$ Kontakte & 20.4 & 20.2 \\
\hline$>2-3$ Kontakte & 17.7 & 17.0 \\
\hline >3-4 Kontakte & 13.6 & 12.9 \\
\hline$>4-6$ Kontakte & 16.9 & 15.7 \\
\hline$>6-8$ Kontakte & 8.1 & 7.6 \\
\hline >8 Kontakte & 7.1 & 6.6 \\
\hline \multicolumn{3}{|c|}{$\begin{array}{l}\text { Alle Informationen aus TK-Routinedaten; }{ }^{1} \text { Personen mit Pflegestufe } 3 \text { waren aus der } \\
\text { Befragung ausgeschlossen, }{ }^{2} \text { Erfasst über die Anzahl der Abrechnungsfälle; }{ }^{3} \text { Erfasst über } \\
\text { die Anzahl der Abrechnungsfälle, exklusive der Abrechnungsfälle von den Facharztgrup- } \\
\text { pen Laboratoriumsmedizin und Pathologie }\end{array}$} \\
\hline
\end{tabular}

\section{Diskussion}

\section{$\nabla$}

Die vorliegenden Ergebnisse zeigen die unterschiedliche Bewertung der interpersonellen und organisatorischen Aspekte der haus- und fachärztlichen Versorgung aus Sicht von chronisch Kranken.

Insgesamt wurden alle abgefragten Aspekte der haus- und fachärztlichen Versorgung überwiegend positiv bewertet. Über alle Aspekte hinweg wurde dabei die hausärztliche Versorgung besser bewertet als die fachärztliche Versorgung. In einer Auswertung der Weissen Liste basierend auf Daten aus Onlinebewertungsportalen zeigte sich ein ähnliches Ergebnis: Hausärzte wurden insgesamt positiver bewertet als Fachärzte [21]. Dabei bestanden jedoch auch große Unterschiede zwischen den einzelnen Facharztgruppen, welche in der vorliegenden Studie nicht analysiert werden konnten. 


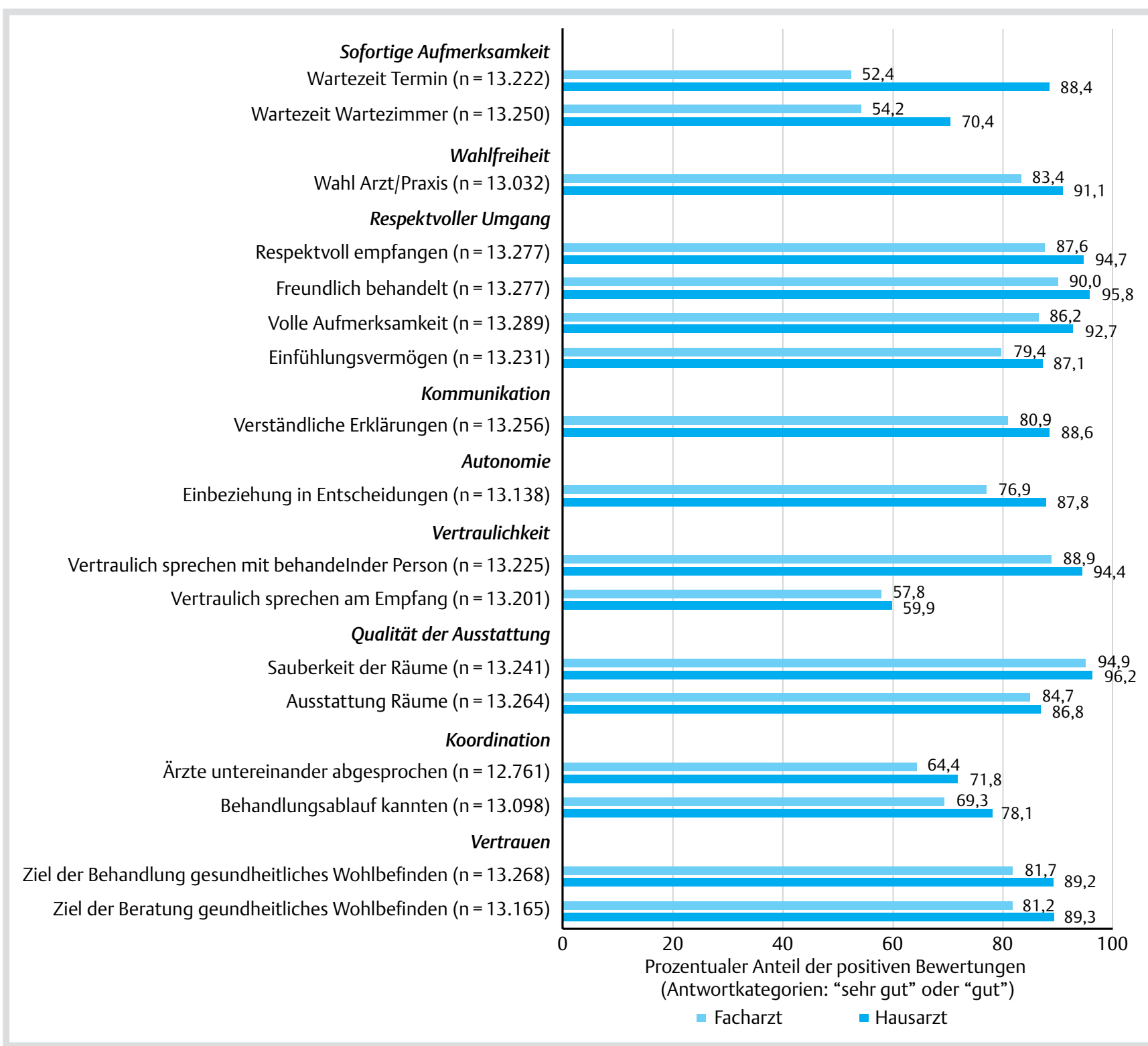

Abb. 2 Bewertung der Responsiveness der haus- und fachärztlichen Versorgung; alle Unterschiede in den Angaben zu haus- und fachärztlicher Versorgung sind auf dem Niveau $p<0,001$ statistisch signifikant.

Bezüglich der hausärztlichen Versorgung wurden in anderen Studien vergleichbare Ergebnisse berichtet. Die Wartezeit im Wartezimmer wurde von 70,4\% der Befragten positiv bewertet und ist somit der am zweitschlechtesten bewertete Aspekt der Versorgung; bei einer Analyse der Europep-Befragungen (European Projekt on Patient Evaluation of General Practice Care) aus dem Jahr 2009 war die Wartezeit im Wartezimmer mit $66,7 \%$ positiven Bewertungen der am schlechtesten bewertete Aspekt [22]. Die Einbeziehung in die Entscheidungsfindung wurde in der Europep-Studie von 83,0\% [22], laut OECD von $87,9 \%$ (14; die OECD greift für die deutschen Daten auf eine Befragung des Commonwealth Fund aus dem Jahr 2010 zurück) und in der vorliegenden Studie von 87,8\% der Befragten positiv bewertet. Das Wissen des Arztes über vorherige Untersuchungen bei vorangegangenen Terminen wurde in der Europep-Studie von 78,3\% positiv bewertet [22]; im Vergleich dazu bewerteten in der vorliegenden Studie 78,1\% das Wissen des Hausarztes über den Behandlungsablauf positiv (69,3\% bewerteten das Wis- sen des Facharztes über den Behandlungsablauf positiv). Ähnliche Ergebnisse hinsichtlich der Koordination der Versorgung wurden auch in einer Befragung des Commonwealth Fund aus dem Jahr 2014 mit Personen im Alter von 65 Jahren und älter berichtet: $31 \%$ der Befragten berichteten, dass der Facharzt den bisherigen Behandlungsablauf nicht kannte bzw. dem Hausarzt relevante Informationen des Facharztes fehlten [23]. Größere Unterschiede zeigten sich bei der Bewertung der Kommunikation: während die OECD 94,7\% positive Bewertungen berichtet [14], lag der Wert in der vorliegenden Studie bei $88,6 \%$. Bei den Vergleichen ist jedoch zu beachten, dass in der vorliegenden sowie der Europep-Studie ausschließlich chronisch kranke Personen befragt wurden, während diese Auswahl bei den OECD-Daten nicht getroffen wurde.

Bei dem berichteten Verzicht auf Versorgung wurde häufiger ein Fach- als ein Hausarzttermin genannt. Von 13685 Befragten gaben 1920 Personen (14,0\%) an, auf medizinische Versorgung verzichtet zu haben. Davon berichteten 1124 Personen den 


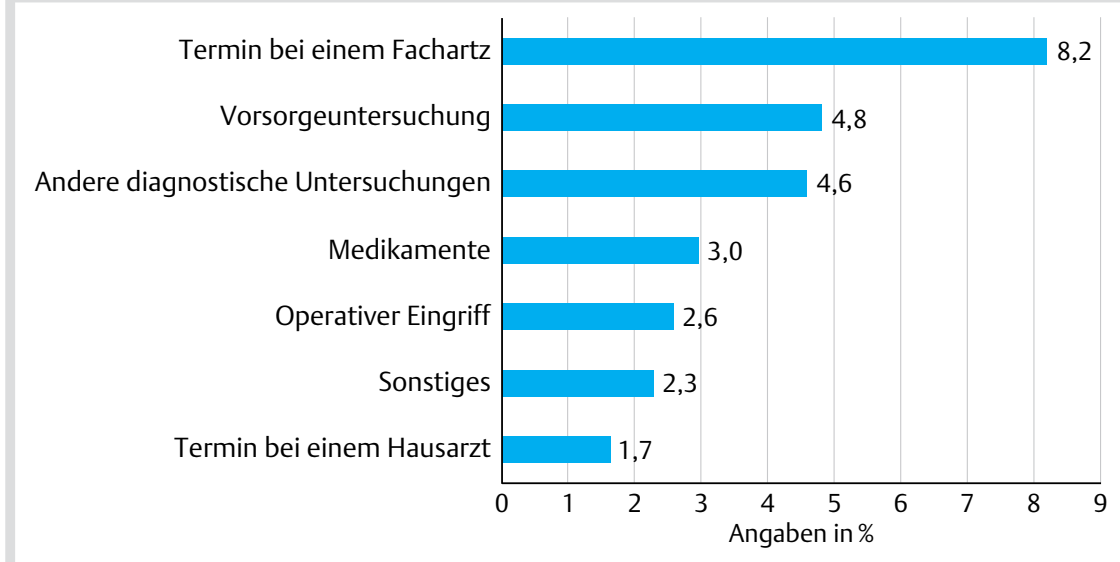

Abb. 3 Anteil der Befragten, die einen Verzicht auf Versorgung berichteten, nach Art des Verzichts $(n=13685)$.

Verzicht auf einen Facharztbesuch. Der Anteil der Befragten, der einen Verzicht auf Versorgung berichtete, ist mit 14,0\% im Vergleich zu anderen Studien relativ hoch. In EU-SILC (European Union Statistics on Income and Living Conditions) 2012 berichteten 94,2\% der Befragten in Deutschland keinen Verzicht auf aus ihrer Sicht notwendige medizinische Versorgung [24]. Hierbei ist jedoch anzumerken, dass zum einen die Stichproben der beiden Studien schwer vergleichbar sind und zum anderen in der EU-SILC Befragung explizit nach einem Verzicht auf Versorgung bei dringender Notwendigkeit gefragt wurde [25]. Diese Formulierung wurde in der vorliegenden Studie bewusst nicht gewählt, um auch einen Verzicht auf z. B. Vorsorgeuntersuchungen erfassen zu können.

In der SHARE Befragung (Survey of Health, Ageing and Retirement in Europe, 1. Welle, 2004) gab ebenfalls ein geringerer Anteil (6,3\% von 2680) der Befragten an, medizinische Versorgung nicht wahrgenommen zu haben. Die Stichproben von SHARE und der vorliegenden Studie sind in Bezug auf die Altersverteilung besser vergleichbar. Bei SHARE wurde jedoch explizit nur nach dem Verzicht auf medizinische Versorgung aus Kostengründen oder mangelnder Verfügbarkeit gefragt. Unterteilt nach den Gründen, gaben 163 Personen (5,5\%) an, aus Kostengründen verzichtet zu haben [26]. In der vorliegenden Studie war der Anteil an Personen, die aufgrund von Kosten verzichtet haben, mit 3\% von 13685 Befragten, wiederum deutlich geringer.

Für die Unterschiede zwischen haus- und fachärztlicher Versorgung bestehen verschiedene Erklärungsansätze. Es kann insbesondere die Annahme getroffen werden, dass der Hausarzt insgesamt häufiger aufgesucht wird und einen wichtigeren Bezugspunkt in der Versorgung darstellt. Somit wird der Hausarzt bei Unzufriedenheit voraussichtlich eher gewechselt als ein Facharzt, welcher weniger häufig aufgesucht wird. Des Weiteren können die Unterschiede in der Bewertung der Versorgung auch durch Unterschiede in der Erwartungshaltung gegenüber Haus- und Fachärzten bedingt sein. Obwohl die gleichen Personen jeweils beide Fragen beantwortet haben, können dennoch Unterschiede in der Erwartungshaltung die Ergebnisse beeinflussen.

Es konnte zudem nicht zwischen verschiedenen Facharztgruppen unterschieden werden, sondern nur allgemein die Gruppe der Fachärzte mit der Gruppe der Hausärzte verglichen werden, sodass Unterschiede zwischen den Facharztgruppen nicht abgebildet werden können. Hinsichtlich der Bewertung der Wartezeiten wurde darüber hinaus nicht zwischen einem Termin mit Überweisung und/oder Terminvereinbarung durch den Hausarzt und die Terminvereinbarung ohne Überweisung unter- schieden. Das Vorgehen bei der Terminvereinbarung hat jedoch potentiell Einfluss auf die Bewertung der Wartezeiten [27].

Die Ergebnisse der Analysen können nur mit Einschränkungen auf andere Patienten übertragen werden, da ausschließlich chronisch Kranke und ausschließlich Versicherte der Techniker Krankenkasse befragt wurden. Bei der Interpretation ist zudem zu beachten, dass durch den großen Stichprobenumfang bereits relativ geringe Unterschiede zu statistisch signifikanten Ergebnissen führen.

Dennoch erlauben die Ergebnisse relevante Schlussfolgerungen. Trotz der überwiegend positiven Bewertungen zeigte sich Verbesserungspotenzial bei beiden Arztgruppen bei den Wartezeiten im Wartezimmer, der Möglichkeit vertraulich am Empfang sprechen zu können sowie der Koordination der Versorgung. In Bezug auf die fachärztliche Versorgung zeigt sich zudem Verbesserungspotenzial bei der Einbeziehung in die Entscheidungsfindung sowie bei der Wartezeit auf einen Termin.

Zudem konnten teilweise starke Unterschiede in der Bewertung der haus- und fachärztlichen Versorgung aufgezeigt werden. Insbesondere in internationalen Vergleichen ist jedoch die Bewertung des ambulanten Sektors häufig ausschließlich auf die hausärztliche Versorgung bezogen (u.a. [14]). Die in dieser Studie aufgezeigten Unterschiede weisen jedoch darauf hin, dass dieses Vorgehen - zumindest für Deutschland - zu einer verzerrten, zu positiven Bewertung des ambulanten Sektors führen kann. Dies sollte somit bei der Bewertung der Leistungsfähigkeit von Gesundheitssystemen sowie bei internationalen Vergleichen ambulanter Versorgung beachtet werden.

Die Ergebnisse zeigen weiteren Forschungsbedarf im Bereich der Leistungsbewertung von Gesundheitssystemen, insbesondere in Hinblick auf die Messung der Nutzerorientierung bzw. der Health System Responsiveness auf. Die Komplexität des Themas der Leistungsbewertung zeigt sich u.a. am Beispiel der Wartezeiten: durch international stark divergierende Zugangsregelungen zur fachärztlichen Versorgung, sind die entsprechenden Ergebnisse international nur eingeschränkt vergleichbar. Die Frage, wie in internationalen Vergleichen eine gute und umfassende Abbildung des deutschen ambulanten Sektors aussehen kann, ohne einen Bereich auszuschließen (wie bisher häufig die fachärztliche Versorgung), benötigt somit weitere Aufmerksamkeit.

Interessenkonflikt: Die Autoren geben an, dass kein Interessenkonflikt besteht. 


\section{Danksagung}

$\nabla$

Das Projekt RAC wird als Teil des Gesundheitsökonomischen Zentrums Berlin (BerlinHECOR) vom Bundesministerium für Bildung und Forschung gefördert (Förderkennzeichen: 01EH1202A). Das Projekt wurde in Kooperation mit dem Wissenschaftlichen Institut der TK für Nutzen und Effizienz im Gesundheitswesen (WINEG) durchgeführt.

\section{Ergänzendes Material \\ $\nabla$}

Tab. 4 finden Sie im Internet unter http://dx.doi.org/ 10.1055/s0041-110669.

\section{Literatur}

1 Murray CJ, Frenk J. A framework for assessing the performance of health systems. Bull World Health Organ 2000; 78: 717-731

2 Sachverständigenrat zur Begutachtung der Entwicklung im Gesundheitswesen 2012. Wettbewerb an der Schnittstelle zwischen ambulanter und stationärer Gesundheitsversorgung. Sondergutachten

3 Smith PC, Mossialos E, Papanicolas I et al. Principles of performance measurement - Introduction. In: Smith PC, Mossialos E, Papanicolas I, Leatherman S, (Hrsg.). Performance measurement for health system improvement. Cambridge: Cambridge University Press; 2009: 3-24

4 Arah OA, Westert GP, Hurst J et al. A conceptual framework for the OECD Health Care Quality Indicators Project. Int J Qual Health Care 2006; 18: 5-13

5 Valentine NB, de Silva A, Kawabata K et al. Health system responsiveness: concepts, domains and operationalization. In: Murray CJL, Evans $D B$, (Hrsg.). Health system performance assessment: debates, methods and empiricism. Geneva: World Health Organization; 2003: 573-596

6 Adesanya T, Gbolahan 0 , Ghannam $O$ et al. Exploring the responsiveness of public and private hospitals in Lagos, Nigeria. J Public Health Res 2012; 1: 2-6

7 Bramesfeld A, Wedegärtner F, Elgeti $\mathrm{H}$ et al. How does mental health care perform in respect to service users' expectations? Evaluating inpatient and outpatient care in Germany with the WHO responsiveness concept. BMC Health Serv Res 2007; 7: 99

8 Jones $A M$, Rice $N$, Robone $S$ et al. Inequality and polarisation in health systems' responsiveness: a cross-country analysis. J Health Econ 2011; 30: 616-625

9 Liabsuetrakul T, Petmanee P, Sanguanchua S et al. Health system responsiveness for delivery care in Southern Thailand. Int J Qual Health Care 2012; 24: 169-175

10 Malhotra C, Do YK. Socio-economic disparities in health system responsiveness in India. Health Policy Plan 2013; 28: 197-205

11 Peltzer K, Phaswana-Mafuya N. Patient experiences and health system responsiveness among older adults in South Africa. Glob Health Action 2012; 5: 1-11
12 Üstün TB, Sonmath C, Abdelhay M, Murray CJL. WHS Collaborating Group. The world health surveys. In: Murray CJL, Evans DB, (Hrsg.). Health system performance assessment: debates, methods and empiricism. Geneva: World Health Organization; 2003: 797-808

13 WHO. WHO Multi-country Survey Study on Health and Responsiveness. http://www.who.int/healthinfo/survey/whspaper37.pdf (letzter Zugriff 24 November 2014)

14 OECD. Patient experience with ambulatory care. http://dx.doi. org/10.1787/health_glance-2013-56-en (letzter Zugriff 24 November 2014)

15 Degen C, Möller D, Schlechter C. Patientenzufriedenheit bei onkologischen Erkrankungen - Eine differenzierte Betrachtung der Sektoren Hausarzt, Facharzt, Krankenhaus und Krankenkasse. Gesundheitswesen 2014; 76: 204-209

16 Schoen C, Osborn R, Squires D et al. New 2011 survey of patients with complex care needs in eleven countries finds that care is often poorly coordinated. Health Aff 2011; 30: 2437-2448

17 Röttger J, Blümel M, Fuchs S et al. Assessing the responsiveness of chronic disease care - Is the World Health Organization's concept of health system responsiveness applicable? Soc Sci Med 2014; 113: 87-94

18 Valentine $N$, Prasad A, Rice $N$ et al. Health systems responsiveness: a measure of the acceptability of health-care processes and systems from the user's perspective. In: Smith PC, Mossialos E, Papanicolas I, Leatherman S, (Hrsg.). Performance measurement for health system improvement. Cambridge: Cambridge University Press; 2009: 138-186

19 Röttger J, Blümel M, Engel S et al.. Exploring health system responsiveness in ambulatory care and disease management and its relation to other dimensions of health system performance (RAC) - study design and methodology. Int J Health Policy Manag 2015; 4: 431-437

20 Charlson ME, Charlson RE, Peterson JC et al. The Charlson comorbidity index is adapted to predict costs of chronic disease in primary care patients. J Clin Epidemiol 2008; 61: 1234-1240

21 Weisse Liste. Pressemitteilung 06.09.2013. Arztvergleich: Patienten sehen deutliche Unterschiede zwischen Fachgruppen. http://www. weisse-liste.de/pressemitteilung-06-09-2013.5578.de.html (letzer Zugriff 9. April 2015)

22 Petek D, Künzi B, Kersnik J et al. Patients' evaluations of European general practice-revisited after 11 years. Int J Qual Health Care 2011; 23: 621-628

23 Osborn R, Moulds D, Squires D et al. International survey of older adults finds shortcomings in access, coordination, and patient-centered care. Health Aff 2014; 33: 2247-2255

24 Eurostat. Self-reported unmet needs for medical examination by sex (total), age (total), detailed reason (no unmet needs to declare) and income quintile (total). http://appsso.eurostat.ec.europa.eu/nui/show. do?dataset $=$ hlth_silc_08\&lang = en (letzer Zugriff 9. April 2015)

25 Statistische Ämter des Bundes und der Länder. Gemeinschaftsstatistik über Einkommen und Lebensbedingungen (EU-SILC). Leben in Europa 2014. Personenfragebogen

26 Mielck A, Kiess $R$, von dem Knesebeck $O$ et al. Association between forgone care and household income among the elderly in five Western European countries - analyses based on survey data from the SHARE-study. BMC Health Serv Res 2009; 9: 52

27 Siciliani L, Moran V, Borowitz M. Measuring and comparing health care waiting times in OECD countries. Health Policy 2014; 118: 292-303 